\title{
Searching of Optimum Characteristics of Multi-Layer Switching Architecture in All-Optical Networks
}

\author{
Motaz Daadoo \\ Department of Computer Systems Engineering \\ Palestine Technical University (Kadoorie) \\ Tulkarm, Palestine \\ motaz_dado@hotmail.com
}

\author{
Yousef Daraghmi \\ Department of Computer Systems Engineering \\ Palestine Technical University (Kadoorie) \\ Tulkarm, Palestine \\ y.awwad@ptuk.edu.ps
}

\begin{abstract}
The growth of Internet increases the range of future services that demand more network capacity and higher data rates. Network and system concepts are evolving accordingly using fiber-optic networks with transmission speeds more than 40 $\mathrm{Gb} / \mathrm{s}$ as the base environment. Therefore, the creation of completely optical networks corresponding equipment is required. One of the basic elements of such network is a switchboard. While designing competitive switchboard, we should consider several services including the possibility of authorization, performance, number of ports, encryption, data compression, class of service $(\mathrm{CoS})$ and quality of service $(\mathrm{QoS})$. The paper proposes a new approach to the construction of switchboards, where the problem of servicing the competitive calls is solved. The basic principle of proposed switchboard construction is the application of multilayered matrix. We performed extensive experiments and found that the optimal number of layers which is required to achieve good results is six layers. The results of using the proposed architecture is improving the efficiency of operation and reducing delay time.
\end{abstract}

Keywords-Optical switching; Wavelength conversion; Wavelength division multiplexing.

\section{INTRODUCTION}

All Optical Networks (AONs) are widely regarded as the ultimate solution to the communication bandwidth needs of future generations of communication networks. Network bandwidth is growing significantly at approximately $40 \%$ per year mainly driven by mobile and cloud technologies. As a result there is an increasing requirement from optical transport networks for additional capacity, higher spectral efficiency and lower cost per bit. Prior studies have indicated that in 2017 , $90 \%$ of the client services would be $10 \mathrm{G}$ or below, while the network line rate has reached $100 \mathrm{G}$ and beyond [1].

Several fundamental studies focused on AONs and highlighted that the important property of AONs is the ability of wave routing, inherent only to this class of optical networks [1]-[7]. AONs are considered to be "transparent" to data format, data wavelength and data protocol. Since the switching is "transparent" to the data packet, the network is called alloptical network, for effective functioning of networks based on AONs required optical switches [2], [3]. One major benefit of using wavelength switching to create optical virtual connections is the possible reduction in equipment costs. Since we do not have to convert this wavelength into electrical form at any intermediate network node, we can reduce the number of receivers and transmitters at these nodes. Removing unnecessary components would allow us either to build a cheaper network with the same throughput, or to increase the capacity for same total cost.

In this paper, we describe a Wavelength Division Multiplexing (WDM) that provides wavelength-transparent data paths between end points. The network is based on a new scheme for switching architecture in AONs using a multilayered switchboard. This paper describes the switch architecture and its individual components, evaluates its performance and Evaluation of switch loading dependence on the number of auxiliary layers of multilayered switching architecture has been performed.

Among the categories of all-optical networks [7]-[14]. the class of transparent AONs is best suited to satisfying the needs of a large network or internetwork. These networks perform no optical-to-electrical conversion of the optical payload within the network, thus preserving transparency. Large all-optical internetworks can be formed by interconnecting multiple alloptical networks. They also lend themselves to hierarchical control and management, an important requirement for the commercial deployment of these networks [6]. Therefore, our switchboard design focuses on providing wavelength transparency for data paths, still allowing dynamic reconfiguration.

However, wavelength switching faces challenges that are: routing or data piping from input port to output port, and disambiguation (or conflicts solution); in case of several packets arrive simultaneously on the same output port. The main contribution of this research is overcoming the aforementioned challenges by proposing the multilayer switching architecture that is able to process competitive packets. The first challenge is solved by conventional methods and by means of switchboard architecture. The latter challenge is solved, as a rule, by means of buffering circuits; it is determined by the features of switchboard architecture. After extensive experiments, we found that the optimal number of layers which is required to achieve good results is six layers. The results of using the proposed architecture is improving the efficiency of operation and reducing delay time. 


\section{RELATED WORK}

\section{A. Optical Switching}

A switch can be abstracted as a device that takes a set of $N$ signal inputs and is able to reproduce them in any permuted order at the output. It is characterized by different parameters such as size, switching time and energy, crosstalk, power dissipation and loss. But in terms of switching function, switches are divided into two types: blocking and nonblocking. A switch is said to be nonblocking if it is capable of realizing every interconnection pattern between the inputs and the outputs. If not, the switch is named as blocking. Nonblocking switches are also divided into two groups: A wide-sense nonblocking switch can connect any unused input to any unused output without rerouting any existing connection. But a strict sense (or strictly) nonblocking switch can connect regardless of the connection rule and algorithm. Also there is a broader class of nonblocking switches called rearrangeably nonblocking switches where rerouting of connections could be done. The basic switch architecture is the $N$ x $N$ crossbar switch. It is also called as a space switch because it separates the signals in space [2], [3].

The crosspoint count of a switch is often used as a measure of its complexity Fig. 1 . Therefore it is desirable to reduce the number of crosspoints $\left(N^{2}\right.$ for $\left.N \times N\right)$. This is usually done by building larger switches from stages of smaller crossbar switches. Architectures also vary according to configurations done by the $2 \times 2$ switches, such as Benes, Spanke, Slepian or Clos but mostly crossbar [4], [5].
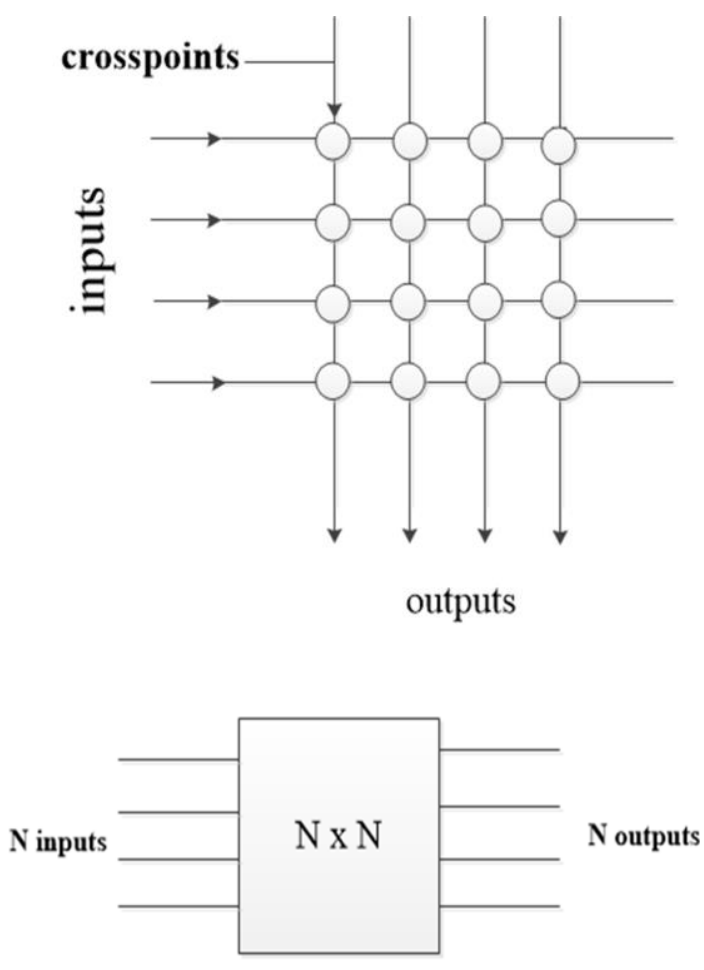

Fig $1 . N$ x $N$ Crossbar switch concept
A crossbar is the ideal and most general form of a switching network. Optical crossbar networks, however, are difficult to scale beyond a small number of ports owing to a number of technological and architectural limitations [6]. Hence, a multistage architecture, consisting of smaller crossbars organized in stages, must be used to attain scalability. In addition, central control of the switching network becomes impractical beyond a certain size; in large networks, it is important to provide the ability to "self-route" data through the network in a distributed fashion using information present in the optical signal itself. Our multistage switch architecture allows such self-routing capability.

Optical switching can be done by the use of one of these architectures but notice that the technology also differs. Optical modulators can be used in different types of technologies and switches are called optomechanical, electrooptic, acoustooptic, magnetooptic, thermooptic or all-optical switches [4], [5].

There is also an important switching architecture used in Wavelength Division Multiplexing (WDM) systems. This switch is known as Optical Cross Connect (OXC) and sometimes called as frequency or wavelength-selective switch. It is composed of multiplexers, demultiplexers and space switch as shown in Fig. 2 [2]. Each of the $N$ input carries $n$ WDM channels. After demultiplexing, the $n N$ channels are switched through a $n N \times n N$ space-division switch. Switch permutes all the channels and then they are multiplexed into $N$ output.

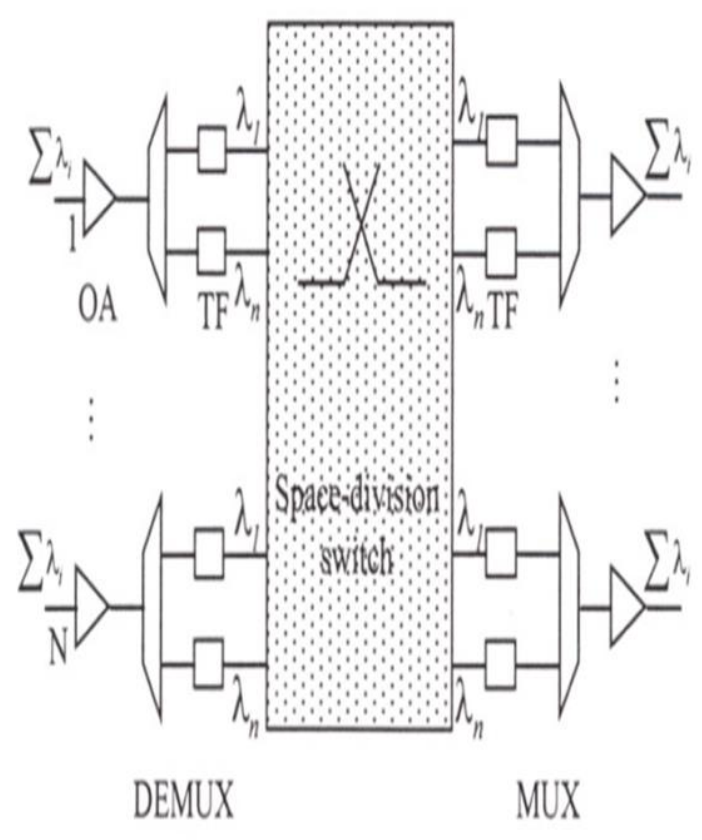

OA=Optical Amplifier, TF= Tunable Filter

Fig 2. Architecture of an optical cross connect 


\section{B. Wavelength Division Multiplexing}

The developments of the fiber optic system initiated wideranging research for optical communication systems. The researchers made innovations in the lasers and in the optoelectronic components as mentioned before. But the increasing demand for bandwidth implies that the capacity of transmission must be increased. In addition, there are two fundamental ways of increasing the capacity: Time Division Multiplexing (TDM) and Frequency Division Multiplexing (FDM) [7].

In the optical domain both are used with optical, means as optical TDM or OTDM and optical FDM or OFDM. One promising method of identifying virtual connections comes through the growing use of Wavelength Division Multiplexing (WDM) over single mode optical fiber as a transmission method. Independent streams of data are modulated using different frequencies and sent through the same piece of fiber. At the receiver, several parallel frequency sensitive filters can be used to separate the signals from each other. Wavelength division multiplexing (WDM) is an emerging technology for increasing the bandwidth of optical networks [10], [12].

In multilayer WDM networks, traffic is carried over optical fiber connections which occupy a wavelength in each traversed fiber and terminates at an optical-to electrical receiver at the destination node [10]-[13]. The connections are optically switched at the intermediate nodes and routing and wavelength assignment mechanisms are drawn on for determining the sequence of optical fibers traversed.

The advent of real-time multimedia services over the Internet has stimulated new technologies for achieving the high level of Quality of Service (QoS) guarantee for sensitive multimedia traffic and for expanding the capacity of optical network backbones [12]. But there is also another types of multiplexing in light wave communications depending on the color (wavelength) of the carrier but different in detection (direct/heterodyne) and separation (optically before/electronically after photo detection) [1]-[9], that is, Dense Wavelength Division Multiplexing (DWDM) is used for the same technique, but when the gaps between adjacent wavelengths are smaller.

To cope up with the increasing high capacity demands, next generation DWDM systems would require line rates greater than $100 \mathrm{~Gb} / \mathrm{s}$. Super-channels are the next-generation technology to increase spectral efficiency and maximize fiber capacity [9]. This will be complimented by the introduction of flexible grid WDM channel plan, to the existing CDC (colorless, directionless and contentionless) architecture of the multi-degree reconfigurable optical add drop multiplexer (ROADM) to become the fundamental building blocks of the next generation DWDM photonic layer [10].

\section{Wavelength Routing Networks}

Wavelength routing networks can be classified either static or reconfigurable depending the elements they contain. If a network does not have any switches on dynamic wavelength converters (described below), it is a static network. Otherwise, it is called reconfigurable or dynamic because of the capability of the network to change routes at nodes [9].
A wavelength converter is an optical device that converts data from one incoming wavelength to another outgoing wavelength. Without wavelength conversion an incoming signal can be optically switched to any output port but only on one wavelength. With wavelength conversion this signal could be optically switched to any output port on any wavelength [10]. Therefore, different physical links can be established where bit rates, protocols become insensitive, thus transparency is provided.

Fig. 3. Shows different types of wavelength conversion[2]. If each wavelength is converted only to itself, then there is no conversion. If each input wavelength is converted to exactly one wavelength, fixed conversion is done. But if each input wavelength can be converted to a specific set of wavelengths, at least one less from all, conversion is named as limited while full conversion implies all possible connections are established.

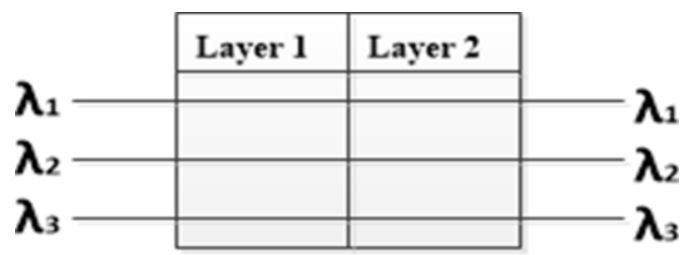

a. No conversion

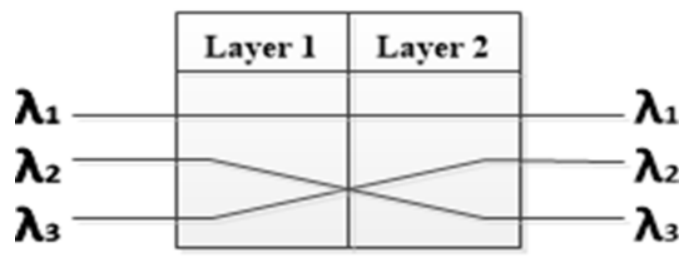

b. Fixed conversion

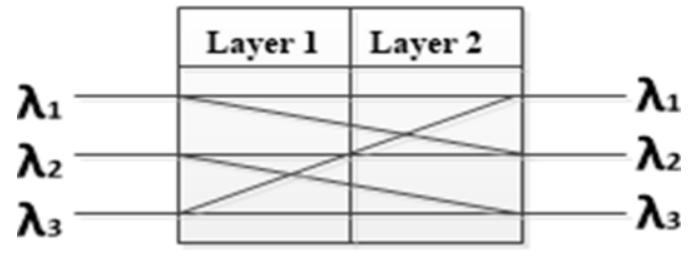

c. Limited conversion

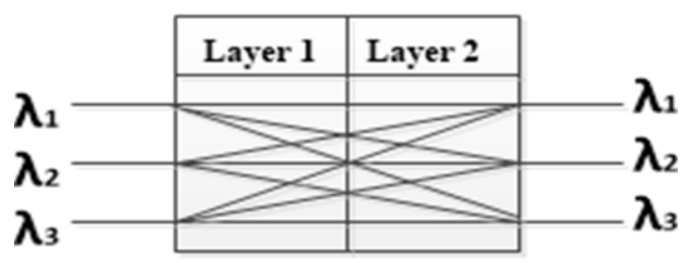

d. Full conversion

Fig 3. Wavelength conversion types 


\section{EVALUATION OF THE SWITCH AS MULTILAYER ARCHITECTURE}

We used the following guidelines in our design of the switchboard architecture:

- The payload must remain in optical form during its passage through the switch cells, that is no opticalelectrical-optical conversion is allowed. This provides complete data-format and wavelength transparency of the signals. However, we do allow for mechanisms to sense the header information from each optical packet, from which the signals for the low-speed electronic control of the path of the optical payload are derived.

- The control function must be distributed within the switch fabric in order to avoid the bottleneck due to a central controller.

- The switchboard architecture must be scalable and modular.

\section{A. Multi-layer Switching Architectures}

In order to construct the switchboard that are able to process the competitive packets, the multilayered switchboard are used as in Fig. 4. Such switchboard improves the efficiency of operation, reduce delay time and decrease their loading.

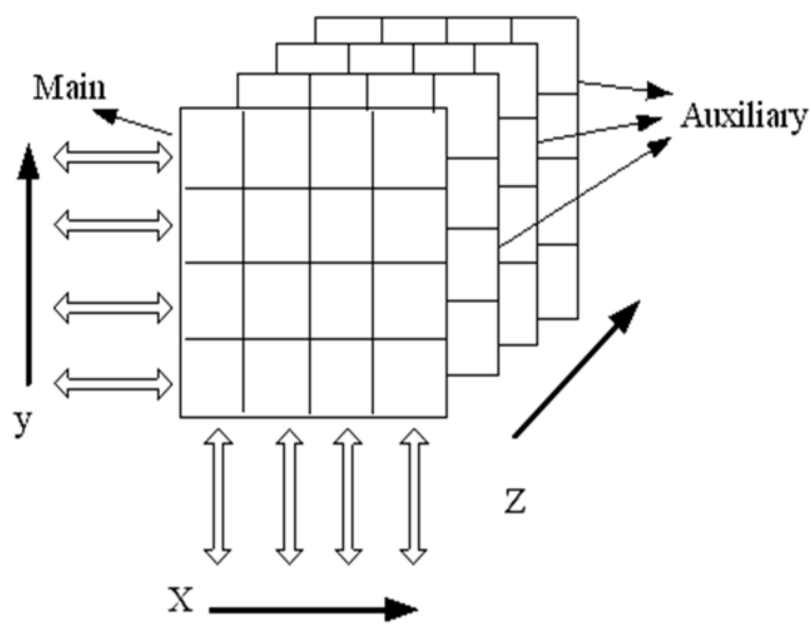

Fig 4. Multi-layered architecture of switching matrix

But in order to apply multilayered switchboard, it is necessary to evaluate the characteristics of its functioning, namely the probability that at certain period of its operation the switchboard will be free or the probability of the freeway in the switchboard for connection of present points [15].

Let the probability of free route from input cell $\left(x_{0}, y_{0}, z_{0}\right)$ to output cell $\left(x_{n_{a}} y_{n}, z_{n}\right)$ be [15]:

$P(A)=P\left(\ldots \begin{array}{c}\left(x_{0}, y_{0}, z_{0}\right):\left(x_{1}, y_{1}, z_{1}\right) ;\left(x_{1}, y_{1}, z_{1}\right):\left(x_{2}, y_{2}, z_{2}\right) ; \ldots \\ \left(x_{n-1}, y_{n-1}, z_{n-1}\right):\left(x_{n}, y_{n}, z_{n}\right) .\end{array}\right)$

where $\mathrm{n}-\mathrm{a}$ number of transfers.

The task is to create a matrix $S(x, y)$ and arrays $L(x, y, z)$ where states of cells are preset, Fig. 5.

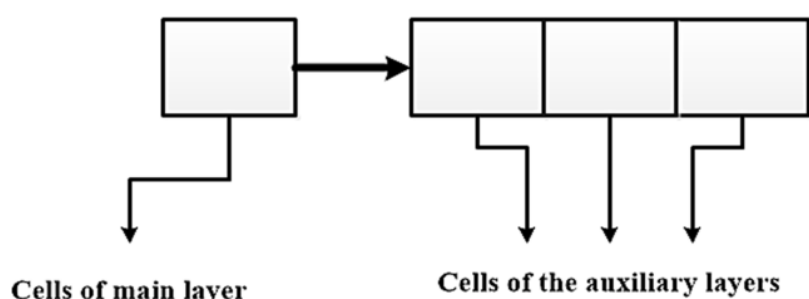

Fig 5. State of occupancy of switch cells

Then the engaged condition is a certain surface below. This surface is conventionally free.

The probability that the route will be free is calculated in the following way:

$$
P(\omega)=1-P\left(S_{1}\right) \cdot P\left(S_{2}\right) \cdot P\left(L_{1}\right) \cdot P\left(L_{2}\right) \cdot P(T)
$$

Where $P\left(S_{1}\right)=P\left(S_{2}\right)=\frac{n \times m}{\text { quantity of engaged }}$ - Probability that selected cell from the main matrix will be engaged $((n \times m)$ size of the basic matrix);

$$
P\left(L_{1}\right)=P\left(L_{2}\right)=\frac{1}{\text { quantity of engaged }} \text { - The probability }
$$

that the selected cell from the line will be engaged ( 1 - length of the line);

$P(T)$ - Probability that the route-making program would find a freeway from the cell $\left(x_{1}, y_{1}, z_{1}\right)$ to the cell $\left(x_{n-1}, y_{n-1}, z_{n-1}\right)$.

\section{B. Switchboard load factor}

If we assume that receiving and processing factor take normal value $(\lambda=0,4$ and $\mu=0,5)$, and the number of switchboard ports equals $m \times n$ and they are within the limits of $16-128$ [15], then switchboard load factor will be determined by expression Fig. 6.

$$
\rho=\frac{\pi}{\mu} \times \frac{1}{\ln (n \times m)}
$$

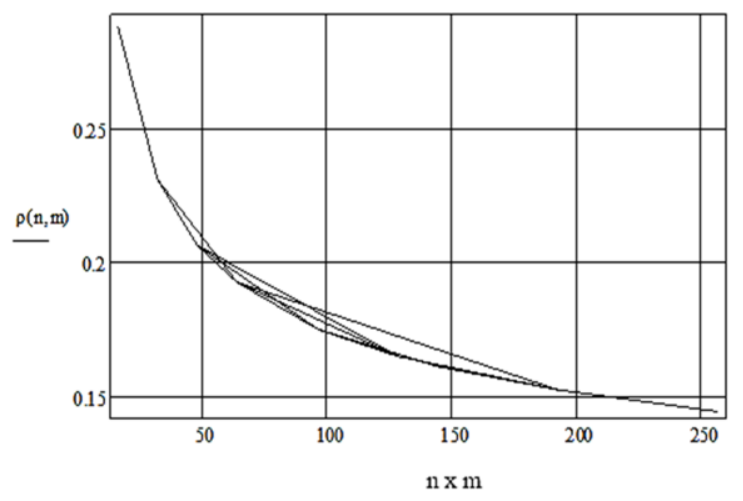

Fig 6.Dependence of switchboard load factor on the sizes of the switchboard $m \times n$ 


\section{Transfer Load}

During the operation of the switchboard the situations will occur; when some cells of basic layer will be engaged. Then, the transfer load will be carried out by auxiliary cells. In this case, the following expression will be valid:

$$
\rho b=\frac{\rho}{k}
$$

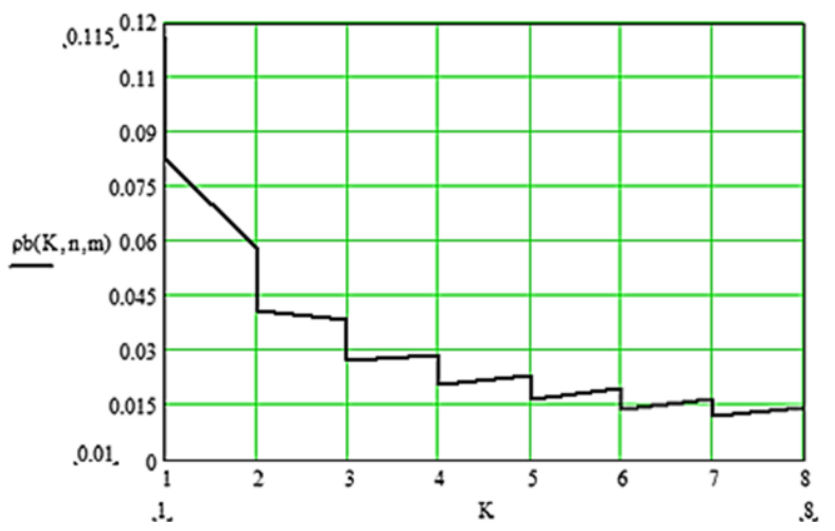

Fig 7. Dependence of switchboard load on quantity of auxiliary layers $(\mathrm{K}=8)$

\section{DIRECTIONS FOR FUTURE RESEARCH}

The payload must remain in optical form during its passage through the switch cells, that is no optical- electrical-optical conversion is allowed. This provides complete data-format and wavelength transparency of the signals. However, we do allow for mechanisms to sense the header information from each optical packet, from which the signals for the low-speed electronic control of the path of the optical payload are derived.

Among the various multistage network architectures [1][13], we chose the class of self-routing switching fabrics, i.e. fabrics that switch incoming signals to the proper destination based on a "routing tag" attached to the payload and is sensed at each switching elements. However, instead of encoding the routing tag as a sequence of bits in a single wavelength channel, we are currently demonstrating the feasibility of routing based on the wavelength-encoded header by constructing a small prototype switchboard. The demonstration of multistage switch fabric is planned for later this year.

\section{CONCLUSION}

On the basis of the suggested material, it can be determined that 6 - layer architecture will be optimal, as it is seen from the graphic Fig 7 the unloading gain of the switchboard considerably decreases. As a matter of fact, for each of switchboard type separate calculations must be carried out and the given version is intended for completely optical switchboard based on 16 - nonblocking ports for 10/100/1000 Ethernet.
While designing competitive switchboard, we should provide such services as the possibility of authorization, performance, number of ports, encryption, data compression, class of service $(\mathrm{CoS})$ and quality of service $(\mathrm{QoS})$, gateway screens, delivery of the detailed information both by the separate user, and by their streams, scalability, security, the opportunity to install additional modules, low price, high degree of readiness, reserve copying and restoration, fault proof.

For further research it should be taken into account that whatever architectural approach is chosen for switchboard design, buffering is required everywhere. Three basic approaches regarding buffers arrangement must be considered Buffering on input, Buffering on output and Buffering inside switching field.

\section{ACKNOWLEDGMENT}

The authors would like to thank a Technical and Applied Research Center (TARC) of the Palestine Technical University - Kadoorie (PTUK) for their support in this work. Our gratitude also goes to all faculty members for their continuous cooperation.

\section{REFERENCES}

[1] S. Roy, A. Malik, A. Deore, S. Ahuja, O. Turkcu, S. Hand, and S. Melle, "Evaluating Efficiency of Multi-Layer Switching in Future Optical Transport Networks," in National Fiber Optic Engineers Conference, 2013, pp. 1-3.

[2] J. Walrand and P. P. Varaiya, High-performance communication networks. Morgan Kaufmann, 2000.

[3] T. X. Brown, "Neural networks for switching," in Neural Networks in Telecommunications, Springer, 1994, pp. 1136.

[4] R. Ramaswami, K. Sivarajan, and G. Sasaki, Optical networks: a practical perspective. Morgan Kaufmann, 2009.

[5] H. Okayama, Y. Okabe, T. Arai, T. Kamijoh, and T. Tsuruoka, "Two-module stage optical switch network," J. Light. Technol., vol. 18, no. 4, 2000, pp. 469-476.

[6] A. Varma and C. S. Raghavendra, Interconnection networks for multiprocessors and multicomputers: theory and practice. IEEE Computer Society Press, 1993.

[7] L. A. Buckman, M. S. Wu, G. Giaretta, G. S. Li, P. Pepeljugoski, G. Jeong, L. Kalampoukas, L. E. Eng, J. W. Goodman, A. Varma, and others, "Demonstration of a Novel All-Optical Switching Node in a Self-Routed Wavelength-Addressable Network (SWANET)," in Proceedings of Optical Fiber Conference, 1995.

[8] L. A. Buckman, "Applications in optical communications: optical transmission of millimeter-wave signals; and, an alloptical wavelength-routed switching network," University of California, Berkeley, 1996.

[9] X. Pang, M. Beltrán, J. Sánchez, E. Pellicer, J. J. Vegas Olmos, R. Llorente, and I. T. Monroy, "Centralized opticalfrequency-comb-based RF carrier generator for DWDM fiber-wireless access systems," J. Opt. Commun. Netw., vol. 6, no. 1, 2014, pp. 1-7.

[10] M. Dzanko, M. Furdek, G. Zervas, and D. Simeonidou, "Evaluating availability of optical networks based on selfhealing network function programmable ROADMs," Opt. Commun. Networking, IEEE/OSA J., vol. 6, no. 11, 2014, pp. 974-987. 
[11] G. Bennett, S. Ahuja, S. J. Hand, C. Liou, V. Vusirikala, and S. Melle, "Analysis of Network Bandwidth Efficiency for Next-Generation 100Gb/s WDM Architectures," in Proc. TERENA Networking Conf, 2010.

[12] A. Amphawan, F. Payne, D. O'Brien, and N. Shah, "Derivation of an analytical expression for the power coupling coefficient for offset launch into multimode fiber," J. Light. Technol., vol. 28, no. 6, 2010, pp. 861-869.

[13] X. Lin, W. Sun, and W. Hu, "Design and Performance of a Cyclic Fault-Tolerant Semiconductor Optical Amplifier Switch Matrix," J. Opt. Commun. Netw., vol. 6, no. 10, 2014, pp. 858-868.

[14] E. Palkopoulou, O. Gerstel, I. Stiakogiannakis, and T. Telkamp, "Impact of IP layer routing policy on multi-layer design," in Optical Fiber Communication Conference, 2014, pp. 1-4.

[15] M. H. Daadoo, G. L. Lysenko, and V. A. Suprigan, "Switching principle with complete passage of cells," Proceeding. Theory and methods of transmission, receiving end processing of information, vol. 3, no. 1, 2004, pp. 57-58. 